\title{
Virtual Reality-Based Treatment Approaches in the Field of Substance Use Disorders
}

\author{
N. Tsamitros ${ }^{1,2}$ (D) M. Sebold ${ }^{2} \cdot$ S. Gutwinski ${ }^{1,2} \cdot$ A. Beck ${ }^{3}$
}

Accepted: 30 April 2021 / Published online: 9 July 2021

(C) The Author(s) 2021

\begin{abstract}
Purpose Substance use disorders (SUD) are burdening chronic conditions characterized by high relapse rates despite severe negative consequences. Substance-related cues that elicit craving by means of automatic physiological and behavioural responses have long been suggested to predict relapse. One major mechanism contributing to relapse behaviour are cue-induced behavioural approach tendencies towards the addictive agent. Recently, there has been an emerging interest in virtual reality (VR)-based approaches to assess and modify craving and its related responses. This review aims at elucidating (1) VR techniques applied in the field of SUD, (2) VR as an induction/assessment tool for biopsychological correlates of craving and (3) VR-based therapeutic approaches.

Findings There is an emerging number of studies focusing on different substances of abuse incorporating VR in craving induction/assessment as well as therapy. Despite some limitations as missing of randomized controlled clinical trials with large samples and missing data on the long-term effects of VR treatment, the VR approach showed consistent results in eliciting and reducing craving across different substances.

Summary This review suggests virtual reality as a promising tool for the assessment and treatment of craving among individuals with substance use disorders. Because of its ecological validity, VR unifies the benefits of a laboratory setting with the advantages of a realistic environment. Further studies with large samples and randomized controlled clinical trials using more homogenous VR techniques as well as assessment of objective biophysiological craving markers are required.
\end{abstract}

Keywords Virtual reality $\cdot$ Addiction $\cdot$ Craving $\cdot$ Cue reactivity $\cdot$ Relapse $\cdot$ Cue exposure therapy $\cdot$ Substance use disorders

\section{Introduction}

Substance use disorders (SUD) are defined as problematic patterns of consumption of psychoactive substances

This article is part of the Topical Collection on New Treatment Approaches in Therapy

N. Tsamitros

nikolaos.tsamitros@charite.de

1 Psychiatrische Universitätsklinik der Charité - Universitätsmedizin Berlin im St. Hedwig Krankenhaus, Institutsambulanz, Berlin, Germany

2 Charité - Universitätsmedizin Berlin, corporate member of Freie Universität Berlin, Humboldt-Universität zu Berlin, and Berlin Institute of Health, Department of Psychiatry and Psychotherapy, Campus Charité Mitte, Berlin, Germany

3 Health and Medical University Potsdam, Faculty of Health, Potsdam, Germany associated with clinical impairment, persistent relapse over time and accompanied with tremendous burden for society and the affected individuals $[1,2]$. As an example, $3.7 \%$ of people living in the European Union and $7.7 \%$ in the USA meet the diagnostic criteria for alcohol dependence with worldwide 3 million deaths every year resulting from harmful alcohol use. Overall, 5.1\% of the global burden of disease and injury as measured in disability-adjusted life years (DALYs) are attributable to alcohol use disorder. Together with relapse rates of up to $85 \%$, this clearly shows the tremendous burden of these diseases and indicates deficits of existing care approaches [3, 4].

Repeated confrontation (exposure) with the substance (ideally in a realistic context), which leads to a reduction in craving, is demonstrably effective in preventing relapse [5]. One major underlying mechanism of exposure therapy is habituation - the decrease of psychobiological increased responses to substance-related stimuli via repeated presentation $[6 \bullet \bullet, 7]$. Moreover, the patient learns alternative behavioural 
strategies in high-risk situations potentially leading to relapse [8]. The higher the variability and dose of the stimuli and presentation contexts, the more effective such therapy is.

At present, however, the use of exposure therapy in the field of substance use disorders has not yet become a routine part of clinical care. The reason for this is that such exposure is hardly feasible in the context of psychotherapeutic practice, as it is associated with an immense time and organizational effort and consequently with high costs [6*0]. Moreover, confrontation with drugs of abuse in daily life encounters in natural settings that substantially differs from the laboratory settings in which exposure therapies have taken place. In addition, the presence of a therapist during exposure therapy may reduce the ability to generalize to situations where no therapist is present $[6 \bullet \cdot$.

Virtual reality cue exposure therapy (VR-CET) allows for numerous advantages over in vivo exposure therapy by (1) significantly increasing practicability, (2) high comparability between different treatment providers through structured and standardized stimulation protocols and (3) high ecological validity combining the benefits of a laboratory setting with the advantages of a realistic environment.

This report aims at reviewing potential benefits as well as pitfalls of the use of virtual reality within the field of SUD. VR has been used in induction, assessment and evaluation of craving and craving-related processes as well as in therapeutic strategies aiming at relapse prevention, and we here provide study results across these domains. Further, we focus our efforts on substance-related addictions; however, research on VR programmes for behavioural addictions has also recently been conducted [9-11].

\section{Introducing Virtual Reality Techniques}

Virtual reality (VR) incorporates a human-computer interaction that goes beyond a conventional desktop. VR systems embed the user in a computer-generated data environment with 3D representation and perception and often spatial interaction in real time [12]. VR hardware and software have been constantly developing since the late 1960s, but lately becoming more popular for commercial, educational, clinical and research applications [13].

Since VR is still a developing medium, definitions are vividly changing. However, state of the art is the definition of four key elements of VR [14] (see Fig. 1):

1) Virtual world as an imaginary space and as a description of objects in a space and the rules and relationships governing these objects

2) (Mental) immersion as a state of being deeply engaged into an alternative reality/environment or point of view and/or as the computer system's technological capacity to deliver a vivid experience that removes the user from physical reality [15]

3) Sensory feedback as an essential ingredient of VR based on the physical positions of the participants (position tracking)

4) Interactivity as the ability of the participant to affect a computer-based world

Another aspect and measure of VR quality related to immersion is the "presence" which refers to the feeling of "being there" in a simulated scene [16]. VR as a term has been used to describe a variety of multimedia technologies including presentations of images and videos on screens, 3D stereoscopic multiwall environments or with head-mounted-displays (HMD) that isolate all external visual stimuli for the user [17]. HMD with motion tracking is becoming the most common medium of VR through ongoing technological and accessibility improvements together with lower costs [18]. Most VR environments focus on visual simulations, but multisensory VR environments with auditory and haptic stimuli are also available, and even simulation of olfactory and gustative senses has been attempted [16]. "Cybersickness" is the most common side effect of VR simulation that is comparable to "motion sickness" from motion environments (e.g. "seasickness" through sea travel) [19].

One important facet in the field of VR application in the healthcare system is the patient perspective on this method. To date, research on the aspect is sparse; however, there is first evidence on positive patients' acceptability that can be achieved using VR. In a study for patients with specific phobias $(n=150), 76 \%$ chose a VR-based exposure over in vivo exposure [20]. A study with patients suffering from PTSD (n = 19) used a VR environment as a tool for coping with negative emotions [21]. The experienced treatment surpassed the expectations of patients that have been already rated as positive prior to treatment. Moreover, a large survey on the public opinion on VR technique application showed positive perceptions about the use of VR in a wide range of healthcare settings [22]. However, some concerns have been expressed regarding radiation, motion sickness and the ubiquity of and overall dependence on technology that should be acknowledged. Clarification, e.g. regarding the non-occurrence of radiation, could be important aspects here.

\section{Introducing Virtual Reality-Based Therapeutic Approaches in the Field of SUD}

In the field of psychiatry, VR-based interventions were originally developed for specific phobia in the 1990s but since then have continuously been adapted to other neurocognitive and psychiatric conditions such as anxiety, post-traumatic 
Fig. 1 The key elements of virtual reality as described by Sherman and Craig (2003)

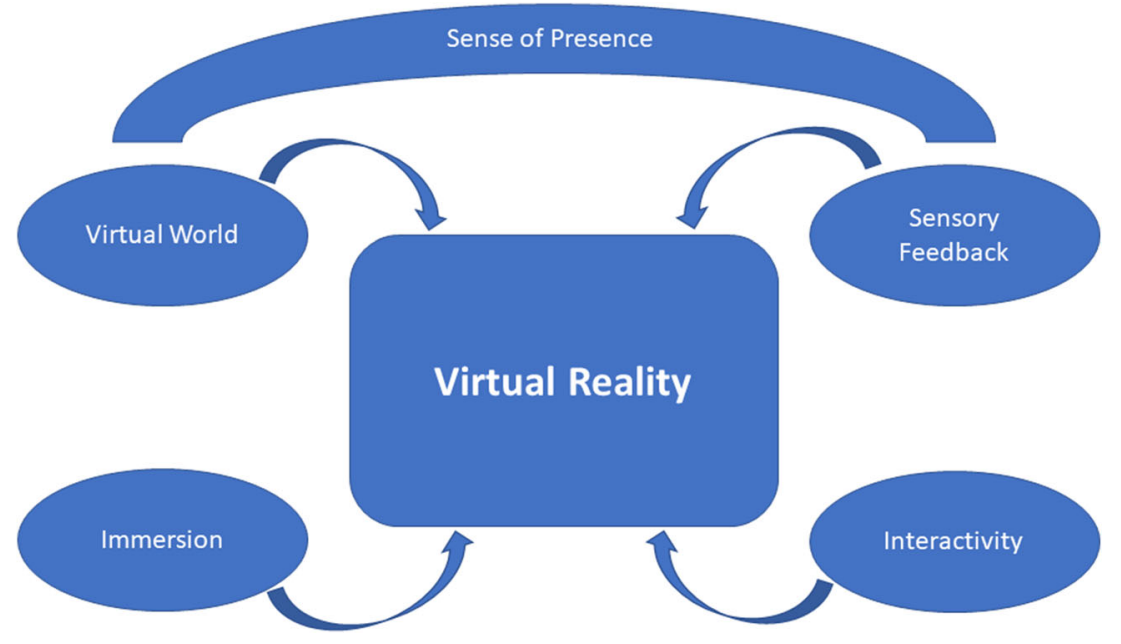

stress and substance use disorders [23, 24]. In most cases, VR therapies consist of elements that are otherwise used in traditional cognitive behavioural approaches, most commonly exposure therapy but also cognitive and behavioural skills training $[23,24]$. To this end, patients can encounter challenging real-world situations in VR that lie at the core of their specific mental health issue and elicit symptoms such as anxiety, fear, paranoia or craving $[25,26]$.

Besides therapeutic VR approaches, there is also a broad range of studies using VR to assess symptoms of the specific disorder. Regarding SUD, VR has been probed to induce craving or to assess cue reactivity, which refers to the physiological and psychological responses to the presentation of substance-related stimuli. However, craving is a multifaceted construct, and previous studies have suggested a mismatch between objective and subjective measures such as physiological parameters and self-reports [27]. To this end, VR is considered a powerful experimental tool to broaden a mechanistic understanding of the craving state, as it allows the collection of both physiological and subjective craving measures in an experimental set-up that mimics reality.

In the following sections, we summarize current studies using VR technique which induce and evaluate craving processes or implement VR as a therapeutic technique. We cluster current research results for different substances of abuse.

\section{Alcohol}

\section{Craving Assessment Via Virtual Reality}

A common procedure for developing ecologically valid VR environments is to conduct exhaustive interviews with studyrelevant individuals. A study by Ghita et al. aimed at determining relevant triggers of alcohol craving in $n=75$ patients with alcohol use disorder (AUD) to develop future VR simulated environments for VR-CET [28]. A variety of situations as well as emotional states seemed to be promising candidates for inducing craving in VR environments. Being at a party, in a restaurant, in a bar or pub and at home as well as emotional states like anxiety or tension, sadness, stress, frustration or irritability were highly associated with urges to drink alcohol. Drinking alone and drinking with two or more friends were equally associated with higher levels of craving. The alcoholic drinks most highly associated with increased levels of craving were beer, wine and whisky. Bordnick et al. assessed alcohol cue reactivity within a novel VR alcohol cue reactivity assessment system incorporating visual, auditory and olfactory stimuli in $\mathrm{n}=40$ non-treatment-seeking drinkers with alcohol use disorders [29]. Overall, subjective craving for alcohol increased across the VR alcohol-related cue environments versus VR neutral cue environments. In line with that, Ryan et al. assessed the ability of VR cue exposure to trigger alcohol craving among students with binge-drinking behaviour ( $\mathrm{n}=$ 15) showing that after VR alcohol exposure students with binge-drinking behaviour reported significantly higher craving for alcohol than students with no binge-drinking behaviour $(n=8)$ [30]. Interestingly, Simon et al. observed that postimmersion craving evoked by a virtual bar was significantly related to the levels of perceived ecological validity of the virtual environment - especially in people with heavy $(\mathrm{n}=$ 18) compared to occasional $(n=22)$ drinking [31].

Cho et al. focussed on the role of social pressure: significantly higher alcohol craving was induced in situations with an avatar (social pressure) than in situations without an avatar (no social pressure) (total sample size $n=10$ ) [32]. In contrast, Lee et al. observed that patients with AUD $(n=14)$ reported extremely high levels of craving immediately upon exposure to a virtual environment with alcohol cues, regardless of social pressure [33•]. In contrast, the craving levels of healthy people with social drinking behaviour $(n=14)$ were influenced by social pressure from virtual avatars.

Kim and Lee developed an approach-avoidance task in a virtual environment to assess the response to virtual alcohol- 
related situations in people with heavy and light social drinking [34]. They observed that the heavy drinking group $(n=18)$ had significantly more difficulties in avoiding alcohol-related situations in the virtual environment than light drinkers $(\mathrm{n}=$ 18) indicating that this virtual approach-avoidance task might be an accurately measure of the levels of social drinkers' craving by allowing individuals to be immersed in virtual environments.

\section{VR-Based Therapeutic Approaches}

Up to now, there are only few studies focussing on VR implementation in therapeutic approaches. In a small sample of eight AUD individuals, Kwon et al. applied CET, using a VR system in eight sessions observing a significant reduction in cue-elicited craving after this VR-CET approach [35]. Similarly, Lee et al. used a VR-CET with multisensory stimulation to reduce craving for alcohol in a small sample of patients with alcohol dependence [32]. In a first study with eight individuals with AUD they showed that VR-CET reduced craving compared to baseline [36]. In a second study, a series of 10 VR-CET sessions compared to the same amount of cognitive behavioural therapy session led to a greater decrease in craving $(n=38)$ [37]. In line with these results, Hyun et al. observed that VR-CET led to a decrease in craving as well as a decrease in brain metabolism as measured with $18 \mathrm{~F}$ fluorodeoxyglucose positron emission tomography after 10 VR-CET sessions compared to baseline in $\mathrm{n}=12$ alcoholdependent subjects [38].

Hernández-Serrano et al. explored potential predictors of changes within craving for alcohol in patients with AUD ( $\mathrm{n}=$ 42) undergoing treatment-as-usual (TAU) or treatment-asusual supplemented with virtual reality cue-exposure therapy [39]. They conclude that VR-CET was beneficial among patients with intense alcohol craving and individuals having used illicit substances prior to treatment.

Choi and Lee used a so-called virtual reality covert sensitization to reduce craving in people with heavy social drinking $(n=20)$ [40]. Here, the effect of an imagery-based aversive treatment (i.e. conditioning via repeatedly pairing heavy drinking with unpleasant aversive stimuli) was enhanced by virtual reality. Indeed - despite a single treatment sessionparticipants changed implicit craving (as assessed via alcohol implicit association test, eye-tracking test and alcohol Stroop test) as well as explicit, self-reported craving. Metcalf et al. developed a serious game in VR for individuals with alcohol and nicotine dependence $(n=61)$ with the aim to strengthen refusal of addiction-related cues [41]. Specifically, users were instructed to hit or kick away drug-related cue images as they fly towards the user. If a user successfully hits the image, it explodes, and the user gains in-game points. The authors reported that craving and substance use decreased, while self- efficacy significantly increased for participants during the intervention period of 4 sessions.

Son et al. conducted a glucose positron emission tomography study before and after VR-CET in a small sample of alcohol-dependent patients $(n=12)$ : compared with the healthy control group, AD subjects showed higher metabolism in the limbic system (i.e. right lentiform nucleus and right temporal lobe) at baseline [42]. After VR-CET, alcoholdependent individuals showed decreased brain metabolism in these two areas relative to that at baseline.

Most recently, Mellentin et al. published a study protocol of a randomized controlled trial to examine the effectiveness of a stimuli-relevant approach-avoidance training programme (AATP) delivered in a VR environment compared to AATP without VR as well as in comparison to treatment as usual (TAU) in a large sample (estimated $n=204$ ) of alcohol use disorder (AUD) patients [43]. The author hypothesized that AATP is more effective than TAU and VR AATP more effective than standard AATP.

Ghita and Gutiérrez-Maldonado summarized in a systematic review that studies using VR in the field of alcohol use disorders present clear limitations: So far, (1) no clinical trials were conducted to explore the efficacy of the VR as a treatment tool; (2) there are no studies on the generalization of craving responses in the real world; or (3) there are no data on the long-term effects of VR treatment [44••].

Despite these limitations, the VR approach showed consistent results as regards eliciting and reducing alcohol craving, suggesting that it is a promising tool for the assessment and treatment of craving among individuals with alcohol misuse.

\section{Nicotine}

\section{Craving Assessment Via Virtual Reality}

García-Rodríguez et al. validated 7 virtual environments for nicotine craving finding the greatest subjective craving increases among participants with nicotine addiction $(\mathrm{n}=46)$ when being in a pub, having a coffee at a cafe or lunch at home although psychophysiological variables as heart rate and electrodermal activity were less sensitive to these changes [45]. A preliminary study with a small size $(n=10)$ of current smokers using a projection wall system as a method of VRCET found a significant increase of psychophysiological responses and subjective craving in a social situation with avatar dialogues (complex cues) as well in a virtual room with object-related cues, while electromyography and electrodermal activity were greater in the first environment [46]. Thomson-Lake et al. found a significant increase of selfreported craving in nicotine-deprived smokers $(n=36)$ being exposed in a VR room with object-related cues and avatar interaction (e.g. cigarettes being offered) compared to 
exposure in a VR-placebo room or compared to exposure in VR room with object-related-cues but without avatar interaction [47]. Notably olfactory cues were also used during exposure in both rooms (but not in placebo). According to the same study, nicotine dependence severity and pre-exposure withdrawal symptoms were both significantly positively correlated with self-reported craving during exposure in the VR room with active avatars.

De Bruijn et al. examined the impact of the degree of immersion and of "presence" during exposure in VR environments without a concluding main effect concerning craving induction [48]. Among current smokers $(n=58)$, craving increased more in a less immersive VR environment. In contrast, among abstinent individuals $(n=30)$, who recently quit smoking, craving increased more in a high-immersive VR environment. Both virtual environments consisted of the same movie in different settings (same 360-degree environment on a smartphone versus VR-headset) with no possibility of user interaction in the VR world. A systematic review by Segawa et al. identified 16 studies $(n=445)$ that reported VR-induced craving in adult smokers [49••]. They concluded that most of the reviewed studies used a single-item analogue scale to assess craving and proposed the use of multidimensional scales. According to the authors, no studies evaluated the effect of different sensorial stimuli used in craving induction, and therefore, further research is needed.

\section{VR-Based Therapeutic Approaches}

Pericot-Valverde et al. exposed treatment-seeking individuals $(\mathrm{n}=48)$ with nicotine dependency in a previously validated VR environment over 5 weeks [45, 50]. Participants treated with weekly VR-CET sessions reported a significant decrease of cue-induced and general craving along with a significant decrease of cigarettes smoked per day. Similarly, in a study by Choi et al. with individuals with nicotine dependence $(n=10)$, the enhanced cue reactivity through exposure to social and object-related cues decreased gradually over a 4-week VRCET [46]. The reduction of cue reactivity was assessed by self-reported craving as well as measures of electromyography and electrodermal activity. Another placebo-controlled study by Culbertson et al. found an enhanced therapeutic effect of smoking VR-CET applied twice a week over an 8-week period regarding quit rate and number of cigarettes smoked per day, with all participants joining a standardized cognitive behavioural therapy $(\mathrm{CBT})$ group [51]. A small sample size $(\mathrm{n}=$ 11) might be a limitation to the findings but a post-hoc withinsubject analysis found a significant decrease in subjective craving during and after each session only for the experimental group but not for the placebo group. Further, individuals with nicotine addiction $(n=60)$ practicing mindfulness during a VR exposure therapy as part of a 21-day multimodal smartphone-based intervention achieved a significant higher abstinence rate at the end of the intervention compared to a control group $(n=60)$ in a randomized controlled trial [52]. This study showed good adherence rates (93\%) for this outpatient treatment group for the use of a VR cardboard headset, which implies generalization to general patient populations.

A different therapeutic approach used a virtual reality game where participants crushed virtual cigarettes with their arm motion [41, 53]. By using this approach, Girard et al. found no significant effect on nicotine abstinence for the cigarettes crushing group $(n=46)$ compared to a placebo virtual condition $(n=45)$ after 4 weeks of treatment [53]. However, they found a significant higher abstinence rate for the cigarettes crushing group at the end of the 12-week programme and at a 6-month follow-up. Explaining these results and considering that participants of the active condition dropped-out later than in the placebo group, the authors suggest that virtual reality tasks might have affected the motivation to longer attend the shared for all participants minimal-support psychosocial treatment programme and increased self-efficacy and self-regulation. A similar theoretical background of these approaches can be found in the related cognitive-bias modification using a computer-based treatment with generally inconsistent results in SUD [54]. Interestingly an upcoming randomized controlled trial $(n=100)$ will incorporate this concept in a VR setting as published in their study protocol by Machulsvbka et al. [55].

\section{Cannabis}

\section{Craving Assessment Via Virtual Reality}

Bordnick et al. found a significant increase of subjective craving and attention to cues for moderate to heavy cannabis smokers $(n=20)$ in VR cannabis environments compared to VR neutral environments [56]. VR cannabis cues included visual, auditory and vibrotactile as well as olfactory and social stimuli.

Up to date, there is no study on VR-based treatment approaches in individuals with cannabis dependence indicating the need for further research.

\section{Cocaine}

\section{Craving Assessment Via Virtual Reality}

Saladin et al. used a VR environment depicting different scenes with actors engaging in crack cocaine-related behaviours (e.g. smoking crack) along with integrated auditory and vibrational stimuli [57]. A small sample $(\mathrm{n}=11)$ of crack cocaine-dependent individuals showed a significant increase of subjective craving and of heart rate variability along with a 
significant decrease of positive affect in the drug content environment compared to a neutral environment. Surprisingly, the scene with the lowest realism rating (using the drug) elicited the strongest craving and heart rate increase, which demonstrates the challenges of designing VR simulations for clinical settings.

Due to the lack of studies examining therapeutical VR approaches in cocaine addiction, further research is needed.

\section{Methamphetamine}

\section{Craving Assessment Via Virtual Reality}

Culbertson et al. used an online gaming platform (Second Life) to create a VR environment with methamphetamine $(\mathrm{METH})$ cues and a neutral VR environment which could be viewed on high-definition screen [58]. A sample of 17 nontreatment seeking METH users reported a significant increase in subjective craving in the METH-VR environment. Additionally, "high craving" participants displayed a higher frequency of cardiovascular activity, while "low craving" participants displayed a lower frequency of cardiovascular activity during all conditions (with METH cues and neutral) suggesting a dichotomy in physiological cue reactivity for patients with METH addiction.

Wang et al. also created a METH-VR environment with emphasis on social cues that induced significant differences of heart rate variability (HRV) between individuals with METH use disorder and healthy controls $(n=61$ vs 45$)$ [59]. Changes in HRV indices such as time domain and non-linear domain were positively correlated with subjective craving but contradicting the findings of Culbertson et al. not frequency domain.

Tsai et al. immersed subjects $(n=20)$ with METH use disorder (MUD) and healthy controls $(n=11)$ in a virtual Karaoke bar using visual and auditory but also flavour stimuli [60•]. Significant differences in physiological measures such as heart rate variability, electrodermal activity and eye tracking measures (dwell times and pupil size) for subjects with MUD but not for controls from pre-VR to post-VR simulation demonstrated a successful craving induction. The exposure included a gradual increase of "temptation" such as asking the participant to read out loud the name of the drug or engaging in drug-related conversations with avatars.

Tan et al. used a VR simulation with METH cues aiming to explore the association between cue reactivity and the circuits of mesocorticolimbic reward and executive control systems in male individuals with METH use disorder $(n=60)$ [61]. Gamma current density measured through EEG with source estimation was decreased in medial prefrontal cortex/ orbitofrontal cortex (MPFC/OFC) and right dorsolateral prefrontal cortex (DLPFC) and predicted changes in skin conductance level that increased significantly through exposure to
METH cues compared to a neutral environment. However, there was no correlation between subjective craving, gamma activity and physiological response indicating the need for further research to advance the understanding of the precise link between self-reports of craving and activity of the autonomous nervous system.

\section{VR-Based Therapeutic Approaches}

Wang et al. developed a VR counterconditioning procedure (VRCP) for patients with METH dependence using VR videos with METH-related cues followed by a second part where the characters in the video experience a distinct adverse consequence caused by METH use (such as being arrested by the police, somatic diseases or even sudden death) [62]. Six VRCP sessions in two studies with large samples (study $1: \mathrm{n}=$ 31 vs 29 , study $2: \mathrm{n}=612$ vs 276 ) resulted in a significant decrease in subjective craving and a more negative attitude towards METH use compared to participants that received treatment as usual. However, willingness to consume the drug was similar between the two groups.

Supporting the theoretical significance of memory reconsolidation in the maintenance of substance use disorder, Liu et al. describe a novel VR procedure in a study protocol for an upcoming randomized controlled trial with four therapeutic groups $(n=95)$ [63]. The authors based on previous results hypothesize that adding a reactivation of drugassociated memories $10 \mathrm{~min}$ to $6 \mathrm{~h}$ prior to exposure may be more effective for reducing craving and cue reactivity than direct exposure. The authors aim to compare effects on craving reduction across a group treated with VR exposure to drug cues (extinction session) $10 \mathrm{~min}$ after an initial session of METH associated memory activation (retrieval session) with a group treated with an extinction session after neutral retrieval. Another group shall be treated with an extinction session after an interval of at least $6 \mathrm{~h}$, while the fourth group will use only video material for retrieval activation.

\section{Conclusion}

The current summary of studies in SUD using VR techniques to either elicit and assess psychophysiological markers of craving or to enhance existing therapeutic approaches clearly suggests that VR is a promising tool for the assessment and treatment of craving among individuals with diverse substance use disorders.

Given the theoretical and practical similarities across CET for SUD and exposure therapy using fear cues in anxiety disorders with the latter being the standard element of CBT, it is worth looking at the progress in virtual exposure therapy in this disorder spectrum [6••]. A systematic review and metaanalysis by Wechsler, Kümpers and Mühlberger comparing 
VR exposure to in vivo exposure for specific phobia, agoraphobia and social phobia demonstrated a wide range of effect sizes, indicating a high potential of VR exposure [64]. The synthesized effect sizes for VR exposure in specific phobia and agoraphobia were non-significant and significant in favour of in vivo exposure in the case of social phobia. Especially, since SUD also is associated with different social aspects (i.e. increased consumption and craving under social pressure [32]), this result might suggest that VR is able to mimic socially difficult situations better than exposure in vivo in a laboratory.

In a three-arm RCT focussing on therapy of social anxiety disorder, VR exposure was superior on the primary outcome measure compared to in vivo exposure with Bouchard et al. discussing the potential effect of the patient-therapist contact during the virtual reality sessions [65]. Gega commenting on this study considers that a mismatch of in vivo and virtual scenarios may even intensify exposure and offer more than real life can [66].

Future research could therefore examine whether implementation of key elements of VET in anxiety disorders could booster effects of new VR/CET protocols for SUD.

As most existing treatment and rehabilitation programmes for SUD are multidimensional, future research is needed to assess which other pharmacological, psychotherapeutic and mutual support treatments are best combined with VR-based therapies. Additionally, the integration of VR-based therapies in early stages of treatment could be an efficient relapse prevention.

All VR systems consist of input devices (sensors and trackers that detect users' actions), output devices (e.g. headmounted displays, HMD) and the VR environment as generated by the hardware [25]. The devices used in the studies we included have been PC-based, smartphone-based or standalone. Currently stand-alone HMD that cost up to a few hundreds of dollars are the leading devices in research as well as in commercial VR use and could be easily available in hospitals and rehabilitation centres.

Up to date, VR has been adopted in the research of SUD, and while diverse application attempts represent different stages of technological and conceptual development, it has the potential to offer efficient clinical tools for the assessment and therapy of substance use disorders. Especially, VR environments that elicit craving have been widely validated for nicotine, alcohol and methamphetamine but also occasionally for cannabis and cocaine.

Measures of biophysiological parameters that objectify craving in VR environments are still inconclusive despite preliminary positive findings, possibly due to methodological difficulties. Here, the application of machine learning techniques might be of special interest, since biophysiological data seem to have a good potential in distinguishing SUD patients from healthy subjects, thus broadening the application of VR as a diagnostic tool $[60 \cdot 67]$.

While advances in VR are technologically driven, previous research primarily focused on examining the effects of VR applications that mostly implement existing assessment and therapy concepts. VR in the field of SUD research has been mostly used as a simulation of real-world situations, but some VR serious games have also been evaluated [41, 53]. The design of new health VR applications requires that biomedical, psychosocial, technological and user experience perspectives must be taken into consideration [68]. Although fully acknowledging the importance of previous findings, when considering VR as a new medium, future interdisciplinary work between VR scientists and SUD researchers could further develop unique applications that maximize the potential of VR.

Funding Open Access funding enabled and organized by Projekt DEAL. $A B$ and $M S$ were in part funded by the Deutsche Forschungsgemeinschaft (DFG, German Research Foundation) (Project-ID 402170461 - TRR 265). The work was further funded by the Cluster of Excellence (DFG, EXC257).

\section{Declarations}

Conflict of Interest All authors declare no competing interests.

Open Access This article is licensed under a Creative Commons Attribution 4.0 International License, which permits use, sharing, adaptation, distribution and reproduction in any medium or format, as long as you give appropriate credit to the original author(s) and the source, provide a link to the Creative Commons licence, and indicate if changes were made. The images or other third party material in this article are included in the article's Creative Commons licence, unless indicated otherwise in a credit line to the material. If material is not included in the article's Creative Commons licence and your intended use is not permitted by statutory regulation or exceeds the permitted use, you will need to obtain permission directly from the copyright holder. To view a copy of this licence, visit http://creativecommons.org/licenses/by/4.0/.

\section{References}

Papers of particular interest, published recently, have been highlighted as:

- Of importance

-• Of major importance

1. APA. Diagnostic and statistical manual of mental disorders DSM-5. 5th ed. Washington. and London: American Psychiatric Publishing; 2013.

2. Auriacombe M, Serre F, Denis C, Fatséas M Diagnosis of addictions. Chapter 10. In: The Routledge Handbook of the Philosophy and Science of Addiction. Routledge; 2018. 
3. World Health Organization, Global status report on alcohol and health 2018. Poznyak V, Rekve D, editors. World Health Organization; 2018.

4. Helzer JE, Robins LN, Taylor JR, Carey K, Miller RH, CombsOrme $\mathrm{T}$, et al. The extent of long-term moderate drinking among alcoholics discharged from medical and psychiatric treatment facilities. N Engl J Med. 1985;312:1678-82.

5. Chambless DL, Ollendick TH. Empirically supported psychological interventions: controversies and evidence. Annu Rev Psychol. 2001;52:685-716.

6.• Byrne SP, Haber P, Baillie A, Giannopolous V, Morley K. Cue exposure therapy for alcohol use disorders: what can be learned from exposure therapy for anxiety disorders? Subst Use Misuse. 2019;54:2053-63 This work describes the core mechanisms of cue exposure and compares CET for anxiety disorders and alcohol use disorders.

7. Beck A, Wüstenberg T, Genauck A, Wrase J, Schlagenhauf F, Smolka MN, et al. Effect of brain structure, brain function, and brain connectivity on relapse in alcohol-dependent patients. Arch Gen Psychiatry. 2012;69:842-52.

8. Rohsenow DJ, Monti PM, Rubonis AV, Gulliver SB, Colby SM, Binkoff JA, et al. Cue exposure with coping skills training and communication skills training for alcohol dependence: 6- and 12month outcomes. Addiction. 2001;96:1161-74.

9. Lee N, Kim J-J, Shin Y-B, Eom H, Kim M-K, Kyeong S, et al. Choice of leisure activities by adolescents and adults with internet gaming disorder: development and feasibility study of a virtual reality program. JMIR Serious Games. 2020;8:e18473. https://doi. org/10.2196/18473.

10. Park SY, Kim SM, Roh S, Soh M-A, Lee SH, Kim H, et al. The effects of a virtual reality treatment program for online gaming addiction. Comput Methods Prog Biomed. 2016;129:99-108.

11. Bouchard S, Robillard G, Giroux I, Jacques C, Loranger C, StPierre $\mathrm{M}$, et al. Using virtual reality in the treatment of gambling disorder: the development of a new tool for cognitive behavior therapy. Front Psychiatry. 2017;8:27.

12. Blach R. Virtual Reality Technology - An Overview. In: Talaba D, Amditis A, editors. Product engineering: tools and methods based on virtual reality. Dordrecht: Springer Netherlands; 2008. p. 21-64.

13. Cipresso P, Giglioli IAC, Raya MA, Riva G. the past, present, and future of virtual and augmented reality research: a network and cluster analysis of the literature. Front Psychol. 2018. https://doi. org/10.3389/fpsyg.2018.02086.

14. Sherman WR, Craig AB, Understanding virtual reality: interface, application, and design. In: The Morgan Kaufmann Series in Computer Graphics; 2018.

15. Oh C, Herrera F, Bailenson J. The effects of immersion and realworld distractions on virtual social interactions. Cyberpsychol Behav Soc Netw. 2019;22:365-72.

16. Scarfe $P$, Glennerster $A$. The science behind virtual reality displays. Annu Rev Vis Sci. 2019;5:529-47.

17. Garrett B, Taverner T, Gromala D, Tao G, Cordingley E, Sun C. Virtual reality clinical research: promises and challenges. JMIR Serious Games. 2018;6:e10839.

18. Jerdan SW, Grindle M, van Woerden HC, Kamel Boulos MN. Head-mounted virtual reality and mental health: critical review of current research. JMIR Serious Games. 2018;6:e14. https://doi.org/ 10.2196/games.9226.

19. Saredakis D, Szpak A, Birckhead B, Keage HAD, Rizzo A, Loetscher T. Factors associated with virtual reality sickness in head-mounted displays: a systematic review and meta-analysis. Front Hum Neurosci. 2020. https://doi.org/10.3389/fnhum.2020. 00096.

20. Garcia-Palacios A, Botella C, Hoffman H, Fabregat S. Comparing acceptance and refusal rates of virtual reality exposure vs. in vivo exposure by patients with specific phobias. CyberPsychol Behav. 2007:10:722-4.

21. Baños RM, Botella C, Guillen V, García-Palacios A, Quero S, Bretón-López J, et al. An adaptive display to treat stress-related disorders: EMMA's World. Br J Guid Couns. 2009;37:347-56.

22. Keller MS, Park HJ, Cunningham ME, Fouladian JE, Chen M, Spiegel BMR. Public perceptions regarding use of virtual reality in health care: a social media content analysis using Facebook. J Med Internet Res. 2017;19:e419. https://doi.org/10.2196/jmir. 7467.

23. Dellazizzo L, Potvin S, Luigi M, Dumais A. Evidence on virtual reality-based therapies for psychiatric disorders: meta-review of meta-analyses. J Med Internet Res. 2020;22:e20889. https://doi. org/10.2196/20889.

24. Emmelkamp PMG, Meyerbröker K. Virtual reality therapy in mental health. Annu Rev Clin Psychol. 2021;17:495-519. https://doi. org/10.1146/annurev-clinpsy-081219-115923.

25. Riva G, Serino S. Virtual reality in the assessment, understanding and treatment of mental health disorders. J Clin Med. 2020. https:// doi.org/10.3390/jcm9113434.

26. Bell IH, Nicholas J, Alvarez-Jimenez M, Thompson A, Valmaggia L. Virtual reality as a clinical tool in mental health research and practice. Dialogues Clin Neurosci. 2020;22:169-77.

27. Grüsser S-M, Heinz A, Raabe A, Wessa M, Podschus J, Flor H. Stimulus-induced craving and startle potentiation in abstinent alcoholics and controls. Eur Psychiatry. 2002;17:188-93.

28. Ghiță A, Teixidor L, Monras M, Ortega L, Mondon S, Gual A, et al. Identifying triggers of alcohol craving to develop effective virtual environments for cue exposure therapy. Front Psychol. 2019. https://doi.org/10.3389/fpsyg.2019.00074.

29. Bordnick PS, Traylor A, Copp HL, Graap KM, Carter B, Ferrer M, et al. Assessing reactivity to virtual reality alcohol based cues. Addict Behav. 2008;33:743-56.

30. Ryan JJ, Kreiner DS, Chapman MD, Stark-Wroblewski K. Virtual reality cues for binge drinking in college students. Cyberpsychol Behav Soc Netw. 2010;13:159-62.

31. Simon J, Etienne A-M, Bouchard S, Quertemont E. Alcohol craving in heavy and occasional alcohol drinkers after cue exposure in a virtual environment: the role of the sense of presence. Front Hum Neurosci. 2020. https://doi.org/10.3389/fnhum.2020.00124.

32. Cho S, Ku J, Park J, Han K, Lee H, Choi YK, et al. Development and verification of an alcohol craving-induction tool using virtual reality: craving characteristics in social pressure situation. CyberPsychol Behav. 2008;11:302-9.

33. Lee JS, Namkoong K, Ku J, Cho S, Park JY, Choi YK, et al. Social pressure-induced craving in patients with alcohol dependence: application of virtual reality to coping skill training. Psychiatry Investig. 2008;5:239-43 This manuscript focusses on the important aspect of social pressure as a trigger/amplifier of craving and the possibility of VR application in social skills training.

34. Kim D-Y, Lee J-H. Development of a virtual approach-avoidance task to assess alcohol cravings. Cyberpsychol Behav Soc Netw. 2015;18:763-6.

35. Kwon H, Choi J, Roh S, Yang BH, Lee JH. Application of virtual reality-cue exposure therapy for reducing alcohol craving. Ann Rev Cyber Ther Telemed. 2006;4:161-6.

36. Lee J-H, Kwon H, Choi J, Yang B-H. Cue-exposure therapy to decrease alcohol craving in virtual environment. CyberPsychol Behav. 2007;10:617-23.

37. Lee SH, Han DH, Oh S, Lyoo IK, Lee YS, Renshaw PF, et al. Quantitative electroencephalographic (qEEG) correlates of craving during virtual reality therapy in alcohol-dependent patients. Pharmacol Biochem Behav. 2009;91:393-7.

38. Hyun SJ, Doug Hyun H, Churl N, Sang Hoon L, Ju Won S. P.6.b.010 Virtual reality therapy decreased metabolism of lentiform 
nucleus in patients with alcohol dependence: PET study. Eur Neuropsychopharmacol. 2013;23:S563.

39. Hernández-Serrano O, Ghiță A, Figueras-Puigderrajols N, Fernández-Ruiz J, Monras M, Ortega L, et al. Predictors of changes in alcohol craving levels during a virtual reality cue exposure treatment among patients with alcohol use disorder. J Clin Med. 2020. https://doi.org/10.3390/jcm9093018.

40. Choi YJ, Lee J-H. The effect of virtual covert sensitization on reducing alcohol craving in heavy social drinkers. Virtual Reality. 2015;19:111-7.

41. Metcalf M, Rossie K, Stokes K, Tallman C, Tanner B. Virtual reality cue refusal video game for alcohol and cigarette recovery support: summative study. JMIR Serious Games. 2018;6:e7. https://doi.org/10.2196/games.9231.

42. Son JH, Lee SH, Seok JW, Kee BS, Lee HW, Kim HJ, et al. Virtual reality therapy for the treatment of alcohol dependence: a preliminary investigation with positron emission tomography/ computerized tomography. J Stud Alcohol Drugs. 2015;76:620-7.

43. Mellentin AI, Nielsen AS, Ascone L, Wirtz J, Samochowiec J, Kucharska-Mazur J, et al. A randomized controlled trial of a virtual reality based, approach-avoidance training program for alcohol use disorder: a study protocol. BMC Psychiatry. 2020;20:340. https:// doi.org/10.1186/s12888-020-02739-1.

44.• Ghiță A, Gutiérrez-Maldonado J. Applications of virtual reality in individuals with alcohol misuse: a systematic review. Addict Behav. 2018;81:1-11 This is the only systematic review on VR application in the field of alcohol use disorder and sheds light on potentials and pitfalls of this method.

45. García-Rodríguez O, Pericot-Valverde I, Gutiérrez-Maldonado J, Ferrer-García M, Secades-Villa R. Validation of smoking-related virtual environments for cue exposure therapy. Addict Behav. 2012;37:703-8.

46. Choi J-S, Park S, Lee J-Y, Jung H-Y, Lee H-W, Jin C-H, et al. The effect of repeated virtual nicotine cue exposure therapy on the psychophysiological responses: a preliminary study. Psychiatry Investig. 2011;8:155-60.

47. Thompson-Lake DGY, Cooper KN, Mahoney JJ, Bordnick PS, Salas R, Kosten TR, et al. withdrawal symptoms and nicotine dependence severity predict virtual reality craving in cigarettedeprived smokers. Nicotine Tob Res. 2015;17:796-802.

48. de Bruijn G-J, de Vries J, Bolman C, Wiers R. (No) escape from reality? Cigarette craving in virtual smoking environments. J Behav Med. 2021;44:138-43.

49.• Segawa T, Baudry T, Bourla A, Blanc J-V, Peretti C-S, Mouchabac $\mathrm{S}$, et al. Virtual reality (VR) in assessment and treatment of addictive disorders: a systematic review. Front Neurosci. 2020;13:1409 This is the most recent systematic review on VR application across addictive disorders.

50. Pericot-Valverde I, Secades-Villa R, Gutiérrez-Maldonado J, García-Rodríguez O. Effects of systematic cue exposure through virtual reality on cigarette craving. Nicotine Tob Res. 2014;16: 1470-7.

51. Culbertson CS, Shulenberger S, De La Garza R, Newton TF, Brody AL. Virtual reality cue exposure therapy for the treatment of tobacco dependence. J Cyber Ther Rehabil. 2012;5:57-64.

52. Goldenhersch E, Thrul J, Ungaretti J, Rosencovich N, Waitman C, Ceberio MR. Virtual reality smartphone-based intervention for smoking cessation: pilot randomized controlled trial on initial clinical efficacy and adherence. J Med Internet Res. 2020;22:e17571. https://doi.org/10.2196/17571.

53. Girard B, Turcotte V, Bouchard S, Girard B. Crushing virtual cigarettes reduces tobacco addiction and treatment discontinuation. CyberPsychol Behav. 2009;12:477-83.

54. Heitmann J, Bennik EC, van Hemel-Ruiter ME, de Jong PJ. The effectiveness of attentional bias modification for substance use disorder symptoms in adults: a systematic review. Syst Rev. 2018;7:160. https://doi.org/10.1186/s13643-018-0822-6.

55. Machulska A, Eiler TJ, Grünewald A, Brück R, Jahn K, Niehaves $\mathrm{B}$, et al. Promoting smoking abstinence in smokers willing to quit smoking through virtual reality-approach bias retraining: a study protocol for a randomized controlled trial. Trials. 2020;21:227. https://doi.org/10.1186/s13063-020-4098-5.

56. Bordnick PS, Copp HL, Traylor A, Graap KM, Carter BL, Walton A, et al. Reactivity to cannabis cues in virtual reality environments. J Psychoactive Drugs. 2009;41:105-12.

57. Saladin ME, Brady KT, Graap K, Rothbaum BO. A preliminary report on the use of virtual reality technology to elicit craving and cue reactivity in cocaine dependent individuals. Addict Behav. 2006;31:1881-94

58. Culbertson C, Nicolas S, Zaharovits I, London ED, De La Garza R, Brody AL, et al. Methamphetamine craving induced in an online virtual reality environment. Pharmacol Biochem Behav. 2010;96: 454-60.

59. Wang Y-G, Shen Z-H, Wu X-C. Detection of patients with methamphetamine dependence with cue-elicited heart rate variability in a virtual social environment. Psychiatry Res. 2018;270:382-8.

60. Tsai M-C, Chung C-R, Chen C-C, et al (2021) An intelligent virtual-reality system with multi-model sensing for cue-elicited craving in patients with methamphetamine use disorder. IEEE Trans Biomed Eng 1-1. In this important and well-designed study multiple machine learning methods showed significant potential in differentiating between methamphetamine users and healthy controls using biophysiological markers assessed during VR cue exposure.

61. Tan H, Chen T, Du J, Li R, Jiang H, Deng C, et al. Drug-related virtual reality cue reactivity is associated with gamma activity in reward and executive control circuit in methamphetamine use disorders. Arch Med Res. 2019;50:509-17.

62. Wang Y, Liu M, Shen Z. A virtual reality counterconditioning procedure to reduce methamphetamine cue-induced craving. J Psychiatr Res. 2019;116:88-94.

63. Liu W, Chen X-J, Wen Y-T, Winkler MH, Paul P, He Y-L, et al. Memory retrieval-extinction combined with virtual reality reducing drug craving for methamphetamine: study protocol for a randomized controlled Trial. Front Psychiatry. 2020. https://doi.org/10. 3389/fpsyt.2020.00322.

64. Wechsler TF, Kümpers F, Mühlberger A. Inferiority or even superiority of virtual reality exposure therapy in phobias? - a systematic review and quantitative meta-analysis on randomized controlled trials specifically comparing the efficacy of virtual reality exposure to gold standard in vivo exposure in agoraphobia, specific phobia, and social phobia. Front Psychol. 2019. https://doi.org/10.3389/ fpsyg.2019.01758.

65. Bouchard S, Dumoulin S, Robillard G, Guitard T, Klinger É, Forget $\mathrm{H}$, et al. Virtual reality compared with in vivo exposure in the treatment of social anxiety disorder: a three-arm randomised controlled trial. Br J Psychiatry. 2017;210:276-83.

66. Gega L. The virtues of virtual reality in exposure therapy. Br J Psychiatry. 2017;210:245-6.

67. Ding X, Li Y, Li D, Li L, Liu X. Using machine-learning approach to distinguish patients with methamphetamine dependence from healthy subjects in a virtual reality environment. Brain Behav. 2020;10:e01814. https://doi.org/10.1002/brb3.1814.

68. Tao G, Garrett B, Taverner T, Cordingley E, Sun C. Immersive virtual reality health games: a narrative review of game design. J Neuroeng Rehabil. 2021;18:31. https://doi.org/10.1186/s12984020-00801-3.

Publisher's Note Springer Nature remains neutral with regard to jurisdictional claims in published maps and institutional affiliations. 